\title{
Editorial
}

\section{Genomics of Atrial Fibrillation and Heart Failure}

Atrial fibrillation (AF), the most common types of arrhythmia in the clinic, is an increasing health burden in developing and develped countries as well. The prevalence of AF increases with age, increasing from $1 \%$ in youth to nearly $10 \%$ in those age over 80 . In the United States (US), 3 million people are affected by AF and in China, 10 million AF patients exists. Interestingly, heart failure (HF) is among the serious complications of $\mathrm{AF}$, making it contributes to public health burden worldwide. Recent insights into the genomics causes of cardiovascular diseases highlighted the importance of single-gene defects and common variants in AF and HF. Besides that, microRNAs (miRNAs) dysregulation has also been implicated in the etiology and pathogenesis of AF and HF. In this issue, a group of scientists provide their insights about genomics of $\mathrm{AF}$ and $\mathrm{HF}$, pointing out that restoration of genes and/or miRNAs expression to normal level may have therapeutic potential for AF and HF.

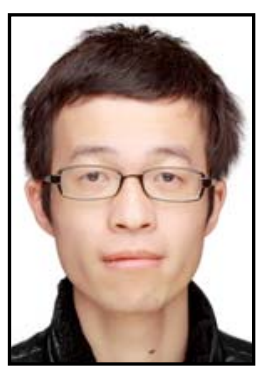

Palatinus et al. summarized the mutations and common variants for $\mathrm{AF}$ while Shen et al. summarized common variants for HF risk and outcome. Both groups discussed these genetic information for the clinic therapy. Hoogen et al. discussed the current knowledge on dysfunction of miRNAs in HF caused by chronic myocarditis, which also provide potential interventions based on miRNAs in the future. Lv et al. overviewed genes and microRNAs responsible for exercise-induced physiological hypertrophy and suggested that manipulation of these critical genes and microRNAs may offer exciting avenues for the treatment of HF.

The collection of articles published in this thematic issue provide the recent progress about the genomic basis for AF and HF. Advances in understanding the genomics of AF and HF will inspaire the interest in facilitating the development of novel genomic based therapies.

Junjie Xiao

Guest Editor

Regeneration and Ageing Lab, School of Life Science

Shanghai University

Shanghai, 200444

China 